\title{
EUCLIDEAN AND CIRCUM-EUCLIDEAN DISTANCE MATRICES: CHARACTERIZATIONS AND LINEAR PRESERVERS*
}

\author{
CHI-KWONG LI $^{\dagger}$, THOMAS MILLIGAN $\ddagger$, AND MICHAEL W. TROSSET ${ }^{\S}$
}

\begin{abstract}
Short proofs are given to various characterizations of the (circum-)Euclidean squared distance matrices. Linear preserver problems related to these matrices are discussed.
\end{abstract}

Key words. Distance geometry, multidimensional scaling, eigenvalues, interlacing inequalities, linear preservers.

AMS subject classifications. 51K05, 15A57, 15A48

1. Introduction. Distance geometry is concerned with the interpoint distances of configurations of $n$ points in metric spaces. It is natural to organize these interpoint distances in the form of an $n \times n$ distance matrix, so the study of distance geometry inevitably borrows tools from matrix theory.

Distance matrices have important applications in a variety of disciplines. Distance matrices were first introduced (anonymously) by A. Cayley [3] in 1841 to derive a necessary condition involving matrix determinants for five points to reside in Eucliden space. Nearly a century after Cayley's contribution, a characterization of distance matrices (re)discovered by G. Young and A.S. Householder [14] was the impetus for (classical) multidimensional scaling [11, 13, 6]. Originally developed by psychometricians and statisticians, multidimensional scaling is a widely used tool for data visualization and dimension reduction. Research on multidimensional scaling continues to exploit facts about distance matrices, e.g., [10]. Analogously, in computational chemistry and structural molecular biology, the problem of determining a

*Received by the editors on Month x, 200x. Accepted for publication on Month y, 200y Handling Editor: .

${ }^{\dagger}$ Department of Mathematics, College of William \& Mary, Williamsburg, VA 23187-8795, USA. ckli@math.wm.edu. Research of this author was supported by a USA NSF grant, a HK RCG grant, and the Key Disciplines of Shanghai Municipality Grant S30104. He is an honorary professor of the University of Hong Kong, the Taiyuan University of Technology, and the Shanghai University.

${ }^{\ddagger}$ Department of Mathematics and Statistics, University of Central Oklahoma, Edmond, OK 73034, USA. tmilligan1@uco.edu. Research of this author was done in part while he was a graduate student at the College of William and Mary with support from USA NSF. He also received support from the Office or Research and Grants at UCO.

$\S^{\S}$ Department of Mathematics, Indiana University, Bloomington, IN 47405 mtrosset@indiana.edu. Research of this author was supported in part by USA NSF, and was done while he was associated with the College of William and Mary. 
molecule's 3-dimensional structure from information about its interatomic distances is the problem of finding a matrix of 3-dimensional Euclidean distances that satisfies certain constraints, as in [4].

It turns out that it is more convenient to encode the squares of the distances between the points, and define a Euclidean (squared) distance (ESD) matrix $A=\left(a_{i j}\right)$ as a matrix for which there exists $x_{1}, \ldots, x_{n} \in \Re^{k}$ such that $a_{i j}=\left\|x_{i}-x_{j}\right\|^{2}$ for a certain positive integer $k$. Let $\mathbf{S}_{n}$ denote the set of $n \times n$ symmetric matrices and let $\operatorname{ESD}(n)$ denote the subset of $\operatorname{ESD}$ matrices. $\operatorname{Then} \operatorname{ESD}(n)$ is a convex cone in $\mathbf{S}_{n}$ with many intriguing properties.

In this paper, we study ESD matrices and circum-Euclidean (squared) distance (CESD) matrices, i.e., $A=\left(\left\|x_{i}-x_{j}\right\|^{2}\right)$ with $\left\|x_{1}\right\|=\cdots=\left\|x_{n}\right\|$. In Section 2, we provide short proofs (some new) of a number of well-known characterizations of ESD matrices. In Section 3, we give characterizations of (CESD) matrices. In Section 4, we characterize linear maps leaving invariant subsets of $\operatorname{ESD}(n)$ and $\operatorname{CESD}(n)$. This can be regarded as a special instance of the general research of linear preserver problems; see [9].

2. Characterizations. Characterizations of distance matrices are mathematically elegant, but also genuinely useful to researchers in other disciplines. In the following, we provide short proofs of several well-known characterizations of Euclidean (squared) distance matrices.

It follows immediately from the definition that an ESD matrix $A=\left(a_{i j}\right)$ with $a_{i j}=\left\|x_{i}-x_{j}\right\|^{2}$, where $x_{1}, \ldots, x_{k} \in \mathbb{R}^{k}$, is a real, symmetric, nonnegative, hollow $\left(a_{i i}=0\right)$ matrix. These properties are necessary but not sufficient for a matrix to be an ESD matrix (a matrix with these properties is called a pre-distance or dissimilarity matrix). If $k$ is the smallest dimension for which such a construction is possible, then $k$ is the embedding dimension of $A$. Furthermore, the choice of $x_{1}, \ldots, x_{n}$ is not unique, for if $\tilde{x}_{i}=x_{i}-x_{0}$ then $\tilde{x}_{i}-\tilde{x}_{j}=x_{i}-x_{j}$. Given $w=\left(w_{1}, \ldots, w_{n}\right)^{t} \in \mathbb{R}^{n}$ with $\sum_{j=1}^{n} w_{j} \neq 0$, let $x_{0}=\sum_{j=1}^{n} w_{j} x_{j} / \sum_{j=1}^{n} w_{j}$. Then $\sum_{j=1}^{n} w_{j} \tilde{x}_{j}=0 \in \mathbb{R}^{k}$, so we can assume without loss of generality that $\sum_{j=1}^{n} w_{j} x_{j}=0 \in \mathbb{R}^{k}$. For notation simplicity, we often assume that $\sum_{j=1}^{n} w_{j}=1$.

Let $e_{1}, \ldots, e_{n}$ denote the coordinate unit vectors in $\mathbb{R}^{n}$ and let $I$ denote the $n \times n$ identity matrix. Set $e=e_{1}+\cdots+e_{n}$ and $J=e e^{t}$. Given $w \in \mathbb{R}^{n}$ such that $e^{t} w=1$, define the linear mapping $\tau_{w}: \mathbf{S}_{n} \rightarrow \mathbf{S}_{n}$ by

$$
\tau_{w}(A)=-\frac{1}{2}\left(I-e w^{t}\right) A\left(I-w e^{t}\right)
$$

Given $w \in \mathbb{R}^{n}$, we say that $x_{1}, \ldots, x_{n} \in \mathbb{R}^{k}$ is $w$-centered if and only if $\sum_{j=1}^{n} w_{j} x_{j}=0$.

Theorem 2.1. Suppose that $A$ is an $n \times n$ real, symmetrix, hollow matrix. Let 
$w$ be any vector in $\mathbb{R}^{n}$ such that $e^{t} w=1$ and let $U$ be any $n \times(n-1)$ matrix for which the $n \times n$ matrix $V=\left(\frac{e}{\sqrt{n}} \mid U\right)$ is orthogonal. Then the following conditions are equivalent.

(a) There exists a w-centered spanning set of $\mathbb{R}^{k},\left\{x_{1}, \ldots, x_{n}\right\}$, for which $A=$ $\left(\left\|x_{i}-x_{j}\right\|^{2}\right)$.

(b) There exists a $w$-centered spanning set of $\mathbb{R}^{k},\left\{x_{1}, \ldots, x_{n}\right\}$, for which $\tau_{w}(A)=$ $\left(x_{i}^{t} x_{j}\right)$.

(c) The matrix $U^{t} A U$ is negative semidefinite of rank $k$.

(d) The submatrix $B$ in

$$
\hat{A}_{0}=\left(\begin{array}{cc}
1 & 0 \\
0 & V^{t}
\end{array}\right)\left(\begin{array}{cc}
0 & e^{t} \\
e & A
\end{array}\right)\left(\begin{array}{cc}
1 & 0 \\
0 & V
\end{array}\right)=\left(\begin{array}{ccc}
0 & \sqrt{n} & 0 \\
\sqrt{n} & * & * \\
0 & * & B
\end{array}\right)
$$

is negative semidefinite of rank $k$.

(e) The matrix $A_{0}=\left(\begin{array}{ll}0 & e^{t} \\ e & A\end{array}\right)$ has has one positive and $k+1$ negative eigenvalues.

(f) There is an $n \times n$ permutation matrix $P$ for which the matrix $\left(\begin{array}{cc}0 & e^{t} \\ e & P^{t} A P\end{array}\right)$ has rank $k+2$, and, for $j=2, \ldots, k+2$, each $j \times j$ leading principal minor is nonzero and has sign $(-1)^{j-1}$.

Proof. We first establish the equivalence of conditions (a), (b), and (c).

$(c) \Rightarrow(b)$. Let $v_{1}, \ldots, v_{n}$ denote the rows of $V$ and let $u_{1}, \ldots, u_{n}$ denote the rows of $U$. It follows from

$$
I=V V^{t}=\left(\begin{array}{cc}
\frac{e}{\sqrt{n}} & U
\end{array}\right)\left(\begin{array}{c}
e^{t} / \sqrt{n} \\
U^{t}
\end{array}\right)=\frac{1}{n} J+U U^{t}
$$

that $U U^{t}=I-\frac{1}{n} J$. Hence, it follows from $J w e^{t}=e e^{t} w e^{t}=e e^{t}=J$ that

$$
U U^{t}\left(I-w e^{t}\right)=\left(I-\frac{1}{n} J\right)\left(I-w e^{t}\right)=\left(I-w e^{t}\right)-\frac{1}{n}(J-J)=\left(I-w e^{t}\right) .
$$

Because $U^{t} A U$ is a negative semidefinite matrix of rank $k$, we can find some $k \times(n-1)$ matrix $Y$ of rank $k$ such that $-\frac{1}{2} U^{t} A U=Y^{t} Y$. Let $W=U^{t}\left(I-w e^{t}\right)$ and let $x_{1}, \ldots, x_{n}$ denote the columns of $X=Y W$. Then

$$
\sum_{j=1}^{n} w_{j} x_{j}=X w=Y U^{t}\left(I-w e^{t}\right) w=Y U^{t}(w-w)=0
$$

and

$$
X^{t} X=W^{t} Y^{t} Y W=-\frac{1}{2} W^{t} U^{t} A U W=-\frac{1}{2}\left(I-w e^{t}\right)^{t} U U^{t} A U U^{t}\left(I-w e^{t}\right)
$$




$$
=-\frac{1}{2}\left(I-e w^{t}\right) A\left(I-w e^{t}\right)=\tau_{w}(A) .
$$

It remains to show that $x_{1}, \ldots, x_{n}$ spans $\mathbb{R}^{k}$. The range space of $U$ is $e^{\perp}$. If $z \in e^{\perp}$, then

$$
\left(I-w e^{t}\right) z=z
$$

hence, $U^{t}$ and $U^{t}\left(I-w e^{t}\right)$ have the same range space. Furthermore, because $Y U^{t}=$ $(0 \mid Y) V^{t}$, rank $Y U^{t}=\operatorname{rank} Y=k$. Hence,

$\operatorname{rank} X=\operatorname{rank} Y W=\operatorname{rank} Y U^{t}\left(I-w e^{t}\right)=\operatorname{rank} Y U^{t}=k$.

(b) $\Rightarrow(a)$. Define $\kappa: \mathbf{S}_{n} \rightarrow \mathbf{S}_{n}$ by

Let $X=\left(x_{1}|\cdots| x_{n}\right)$ and

$$
H=\kappa\left(X^{t} X\right)=\left(x_{i}^{t} x_{i}-2 x_{i}^{t} x_{j}+x_{j}^{t} x_{j}\right)=\left(\left\|x_{i}-x_{j}\right\|^{2}\right) .
$$

Because $J\left(I-w e^{t}\right)=J-J=0$ and $X\left(I-w e^{t}\right)=X-X w e^{t}=X$,

$$
\tau_{w}(H)=-\frac{1}{2}\left(I-e w^{t}\right)\left(D J-2 X^{t} X+J D\right)\left(I-w e^{t}\right)=X^{t} X=\tau_{w}(A)
$$

Furthermore, it follows from (2.2) that

$$
\left(I-w e^{t}\right)\left(e_{i}-e_{j}\right)=e_{i}-e_{j},
$$

so

$$
\begin{aligned}
H_{i j} & =-\frac{1}{2}\left(e_{i}-e_{j}\right)^{t} H\left(e_{i}-e_{j}\right)=\left(e_{i}-e_{j}\right)^{t} \tau_{w}(H)\left(e_{i}-e_{j}\right) \\
& =\left(e_{i}-e_{j}\right)^{t} \tau_{w}(A)\left(e_{i}-e_{j}\right)=A_{i j},
\end{aligned}
$$

i.e., $H=A$.

Notice that this argument demonstrates that $\tau_{w}$ is injective on the hollow symmetric matrices.

(a) $\Rightarrow(c)$. Let $x_{0}=\sum_{j=1}^{n} x_{j} / n$ and $\tilde{x}_{i}=x_{i}-x_{0}$, so that $\tilde{x}_{1}, \ldots, \tilde{x}_{n}$ is an $e$-centered spanning set of $\mathbb{R}^{k}$ with $A=\left(\left\|\tilde{x}_{i}-\tilde{x}_{j}\right\|^{2}\right)$. Let $\tilde{X}$ denote the $k \times n$ matrix with columns $\tilde{x}_{1}, \ldots, \tilde{x}_{n}$. Then $\tilde{X} e=0$, so $\tilde{X} V=(0 \mid \tilde{X} U)$ and it follows that $\operatorname{rank} \tilde{X} U=\operatorname{rank} \tilde{X}=k$.

Because $V$ is orthogonal, $U^{t} e=0$ and therefore $U^{t} J=U^{t} e e^{t}=0=J U$. Applying (2.4),

$$
U^{t} A U=U^{t}\left(J D-2 \tilde{X}^{t} \tilde{X}+D J\right) U=-2 U^{t} \tilde{X}^{t} \tilde{X} U=-2(\tilde{X} U)^{t}(\tilde{X} U)
$$

is a negative semidefinite matrix of rank $k$. 
$(c) \Leftrightarrow(d)$. Because $U^{t} e=0$,

$$
\begin{aligned}
\hat{A}_{0} & =\left(\begin{array}{cc}
1 & 0 \\
0 & V^{t}
\end{array}\right)\left(\begin{array}{ll}
0 & e^{t} \\
e & A
\end{array}\right)\left(\begin{array}{ll}
1 & 0 \\
0 & V
\end{array}\right)=V_{0}^{t} A_{0} V_{0} \\
& =\left(\begin{array}{cc}
1 & 0 \\
0 & e^{t} / \sqrt{n} \\
0 & U^{t}
\end{array}\right)\left(\begin{array}{ll}
0 & e^{t} \\
e & A
\end{array}\right)\left(\begin{array}{ccc}
1 & 0 & 0 \\
0 & e / \sqrt{n} & U
\end{array}\right) \\
& =\left(\begin{array}{ccc}
0 & \sqrt{n} & 0 \\
\sqrt{n} & e^{t} A e / n & * \\
0 & * & U^{t} A U
\end{array}\right) .
\end{aligned}
$$

Thus, $B=U^{t} A U$ and conditions (c) and (d) are equivalent.

Now we establish the equivalence of conditions (d), (e), and (f).

$(d) \Rightarrow(e)$. Because $V$ is orthogonal, so is $V_{0}$ and it follows from (2.5) that $A_{0}$ and $\hat{A}_{0}$ have the same eigenvalues. By interchanging the first two rows of $\hat{A}_{0}$ and performing Gaussian elimination, we see that rank $A_{0}=\operatorname{rank} \hat{A}_{0}=2+\operatorname{rank} B=2+k$. Because $\left(\begin{array}{ll}0 & 0 \\ 0 & B\end{array}\right)$ has no positive eigenvalues and is a principal submatrix of $\hat{A}_{0}$, it follows from the interlacing inequalities that $\hat{A}_{0}$, hence $A_{0}$, has at most one positive eigenvalue. But the leading $2 \times 2$ principal submatrix of $A_{0}$ has a negative determinant and therefore one positive and one negative eigenvalue; hence, by the interlacing inequalities, $A_{0}$ has at least one positive eigenvalue. Thus, $A_{0}$ has exactly one positive eigenvalue and, because rank $A_{0}=k+2, k+1$ negative eigenvalues.

(e) $\Rightarrow(d)$. We have already argued that $\operatorname{rank} B=\operatorname{rank} \hat{A}_{0}-2=\operatorname{rank} A_{0}-2=$ $k+2-2=k$. Given $v \in \mathbb{R}^{n-1}$, we demonstrate that $b=v^{t} B v \leq 0$. Toward that end, let

$$
D=\left(\begin{array}{ccc}
0 & 1 & 0 \\
1 & 0 & 0 \\
0 & 0 & v^{t}
\end{array}\right) \hat{A}_{0}\left(\begin{array}{ccc}
0 & 1 & 0 \\
1 & 0 & 0 \\
0 & 0 & v
\end{array}\right)=\left(\begin{array}{ccc}
0 & \sqrt{n} & 0 \\
\sqrt{n} & c & * \\
0 & * & b
\end{array}\right),
$$

where $c=e^{t} A e / n$. Notice that $\operatorname{det}(D)=-n b$.

Let $d_{1} \geq d_{2} \geq d_{3}$ denote the eigenvalues of $D$. Let $Q$ be any orthogonal matrix of the form

$$
Q=\left(\begin{array}{ccc|c}
0 & 1 & 0 \\
1 & 0 & 0 & * \\
0 & 0 & v &
\end{array}\right)
$$


in which case $Q^{t} \hat{A}_{0} Q=\left(\begin{array}{cc}D & * \\ * & *\end{array}\right)$ has the same eigenvalues as $\hat{A}_{0}$, i.e., the same eigenvalues as $A_{0}$. Because $D$ is a principal submatrix of $Q^{t} \hat{A}_{0} Q$, it follows from the interlacing inequalities that $d_{3} \leq d_{2} \leq 0$. Furthermore, it follows from the RayleighRitz Theorem that $d_{1} \geq 0$. We conclude that $b=-\operatorname{det}(D) / n=-d_{1} d_{2} d_{3} / n \leq 0$.

$(e) \Rightarrow(f)$. Any matrix of the form

$$
\left(\begin{array}{cc}
0 & e^{t} \\
e & P^{t} A P
\end{array}\right)
$$

where $P$ is an $n \times n$ permutation matrix, must have the same eigenvalues as $A_{0}$. It follows from (e) that any such matrix must have rank $k+2$. We choose $P$ so that, for $j=2, \ldots, k+2$, the $j \times j$ leading principal submatrices of (2.6) have no zero eigenvalues. Then the $2 \times 2$ leading principal submatrix is $\left(\begin{array}{ll}0 & 1 \\ 1 & 0\end{array}\right)$, which has a positive eigenvalue. Hence, for $j=2, \ldots, k+2$, each $j \times j$ leading principal submatrix will have one positive and $j-1$ negative eigenvalues and the corresponding minors will have signs $(-1)^{j-1}$.

$(f) \Rightarrow(e)$. Because (2.6) has rank $k+2$, so does $A_{0}$. Because the $2 \times 2$ leading principal minor of (2.6) is negative, the $2 \times 2$ leading principal submatrix has one positive and one negative eigenvalue. Because the $3 \times 3$ leading principal minor of (2.6) is positive, it follows from the interlacing inequalities that the $3 \times 3$ leading principal submatrix has one positive and two negative eigenvalues. Continuing in this manner, we conclude that the $(k+2) \times(k+2)$ leading principal submatrix, hence (2.6), hence $A_{0}$, has one positive and $k+1$ negative eigenvalues.

Let us make some remarks about the characterizations established in Theorem 2.1. We have already noted that the requirement that $x_{1}, \ldots, x_{n}$ is $w$-centered entails no loss of generality; hence, condition (a) is simply the definition of a $k$-dimensional ESD matrix, i.e., an ESD matrix with embedding dimension $k$. Historically, condition (f) was the first alternate characterization discovered. Condition (b) is useful in finding a set of points satisfying the distance matrix. Theoretically, condition (c) is most useful. In the literature, it is sometimes stated slightly differently.

REMARK 2.2. In Theorem 2.1, the statement in condition (c) that $U^{t} A U$ is negative semi-definite can be restated as $\sum_{i, j=1}^{n} a_{i j} y_{i} y_{j} \leq 0$ whenever $\sum_{i=1}^{n} y_{i}=0$, with equality whenever $y$ is in some $n-1-k$-dimensional subspace of $e^{\perp}$.

Proof. Note that $e^{t} y=\sum_{i=1}^{n} y_{i}=0$ is equivalent to $y=U x$ for some $x \in \mathbb{R}^{n-1}$. Hence $\sum_{i, j=1}^{n} a_{i j} y_{i} y_{j}=y^{t} A y=x^{t} U^{t} A U x \leq 0$ exactly when $U^{t} A U$ is negative semidefinite. Note that we can replace the inequality with an equality exactly when $x$ is in 
the $n$ - $1-k$-dimensional null space of $U^{t} A U$, hence $y$ is in some $n-1-k$-dimensional subspace of $e^{\perp}$.

Using either statement for condition (c), we see that $\operatorname{ESD}(n)$ is a convex cone in $\mathbf{S}_{n}$, with embedding dimension increasing up to $n$. It is not easy to check this only using the definition of ESD matrices. Also, from conditions (c) and (e), we can easily deduce the possible ranks of an ESD matrix.

Corollary 2.3 (Gower [7]). If $A \in \operatorname{ESD}(n)$ has embedding dimension $k$, then $\operatorname{rank}(A)$ equals $k+1$ or $k+2$.

Proof. It follows from Theorem 2.1 (c) that $U^{t} A U$ has $k$ negative eigenvalues. Because $U^{t} A U$ is a submatrix of $V^{t} A V$, it follows from the interlacing inequalities that $V^{t} A V$, hence $A$, has at least $k$ negative eigenvalues. Furthermore, because $\operatorname{trace}(A)=0, A$ has at least one positive eigenvalue. Hence, $\operatorname{rank}(A)$ is at least $k+1$. Finally, it follows from (e) that $\operatorname{rank}\left(A_{0}\right)=k+2$. Because $A$ is a submatrix of $A_{0}$, $\operatorname{rank}(A)$ is at most $k+2$.

Gower [7, Theorem 6] distinguished between these two possible cases by demonstrating that $\operatorname{rank}(A)=k+1$ if and only if the points that generate $A$ lie on a sphere. We will give a proof of this fact and some related results in the next section.

3. Circum-Euclidean Squared Distance Matrices. A matrix $A \in E S D(n)$ is CESD if $A=\left(\left\|x_{i}-x_{j}\right\|^{2}\right)$ such that all $\left\|x_{1}\right\|=\cdots=\left\|x_{n}\right\|$. Recall that $C$ is a correlation matrix if $C$ is a positive semidefinite matrix with all diagonal entries equal to one. We have the following characterization of CESD matrices, and the minimum $r$ such that $A=\left(\left\|x_{i}-x_{j}\right\|^{2}\right)$ with $r=\left\|x_{1}\right\|=\cdots=\left\|x_{n}\right\|$. Its proof depends heavily on the canonical form of $A$ in (c).

Theorem 3.1. Suppose that $A \in \operatorname{ESD}(n)$ has embedding dimension $k$. Then the following are equivalent:

(a) $A$ is a CESD matrix.

(b) There exist $\lambda \geq 0$ and a correlation matrix $C$ such that $A=\lambda\left(e e^{t}-C\right)$, equivalently, $\lambda e e^{t}-A$ is positive definite.

(c) The intersection of the null space of $\left(I-\frac{J}{n}\right) A\left(I-\frac{J}{n}\right)$ and the range space of $A$ has dimension one.

(d) Let $V=\left(\frac{e}{\sqrt{n}} \mid U\right)$ be an orthogonal matrix and

$$
V^{t} A V=\left(\begin{array}{cc}
\operatorname{tr} B & v^{t} \\
v & -B
\end{array}\right)
$$

Then there exists a vector $z \in \mathbb{R}^{n-1}$ such that $v=B z$, i.e., $v$ lies in the column space of $B$.

(e) $\operatorname{rank}(A)=k+1$. 
(f) $\sup \left\{w^{t} A w: e^{t} w=1\right\}<\infty$.

(g) There is $w \in \mathbb{R}^{n}$ and $\beta \in \Re$ with $e^{t} w=1$ such that $A w=\beta e$.

(h) Let $V=\left(\frac{e}{\sqrt{n}} \mid U\right)$ be an orthogonal matrix such that

$V^{t} A V=\left(\begin{array}{c|ccc|ccc}b_{0} & b_{1} & \cdots & b_{k} & b_{k+1} & \cdots & b_{n-1} \\ \hline b_{1} & -\lambda_{1} & & & & & \\ \vdots & & \ddots & & & 0 & \\ b_{k} & & & -\lambda_{k} & & & \\ \hline b_{k+1} & & & & & & \\ \vdots & & 0 & & & 0 & \end{array}\right)$,

$$
b_{0}=\sum_{j=1}^{k} \lambda_{j} \text {. Then } b_{k+1}=\cdots=b_{n-1}=0 .
$$

(i) There exists an $M$ such that $\sum_{i, j=1}^{n} a_{i j} y_{i} y_{j} \leq M\left(\sum_{i=1}^{n} x_{i}\right)^{2}$ for all $y \in \mathbb{R}^{n}$.

Moreover, if these equivalent conditions hold and if $A=\left(\left\|x_{i}-x_{j}\right\|^{2}\right)$ with $r=\left\|x_{1}\right\|=$ $\cdots=\left\|x_{n}\right\|$, then

$$
2 n r^{2} \geq \operatorname{tr} B+\left(v^{t} B^{-1} v\right)^{1 / 2}=\left(b_{0}+\sum_{j=1}^{k} b_{k}^{2} / \lambda_{j}\right) / n,
$$

where $B$ is the matrix in condition (d), and $b_{0}, b_{1}, \ldots, b_{k}, \lambda_{1}, \ldots, \lambda_{j}$ are the quantities in (c).

Proof. We prove the equivalence of these all but the last statement by pairs.

(a) $\Leftrightarrow(b)$. If $A=\left(\left\|x_{i}-x_{j}\right\|^{2}\right)$ such that $r=\left\|x_{1}\right\|=\cdots=\left\|x_{n}\right\|$, then

$$
A=\left(\left\|x_{i}\right\|^{2}+\left\|x_{j}\right\|^{2}-2 x_{i}^{t} x_{j}\right)=\lambda\left(e e^{t}-C\right),
$$

where $\lambda=2 r^{2}$ and $C=\left(x_{i}^{t} x_{j}\right) / r^{2}$ is a correlation matrix.

Conversely, if $A=\lambda\left(e e^{t}-C\right)$, where $C$ is a correlation matrix. Then $C=\left(y_{i}^{t} y_{j}\right)$ for some unit vectors $y_{1}, \ldots, y_{n}$. Let $x_{i}=\sqrt{\lambda / 2} y_{i}$ for $i=1, \ldots, n$. Then $A=$ $\left(\left\|x_{i}-x_{j}\right\|^{2}\right)$.

(b) $\Leftrightarrow(h)$. Suppose $V$ is such that $V^{t} A V$ has the form (3.1). Because $\Lambda=U^{t} A U$ is negative semidefinite of $\operatorname{rank} k, \lambda_{1}, \ldots, \lambda_{k}>0$. 
Suppose (b) holds. Writing $C=e e^{t}-A / \lambda$, it follows from (3.1) that

$$
V^{t} C V=\frac{1}{\lambda}\left(\begin{array}{c|ccc|ccc}
n \lambda-b_{0} & -b_{1} & \cdots & -b_{k} & -b_{k+1} & \cdots & -b_{n-1} \\
\hline-b_{1} & \lambda_{1} & & & & & \\
\vdots & & \ddots & & & 0 & \\
-b_{k} & & & \lambda_{k} & & & \\
\hline-b_{k+1} & & & & & & \\
\vdots & & 0 & & & 0
\end{array}\right) .
$$

Because $\lambda C$, hence $V^{t} C V$, is positive semidefinite, so are the principal submatrices

$$
\left(\begin{array}{cc}
n \lambda-b_{0} & -b_{i} \\
-b_{i} & 0
\end{array}\right)
$$

which necessitates $b_{i}=0$ for $i=k+1, \ldots, n-1$.

Conversely, suppose $b_{k+1}=\cdots=b_{n-1}=0$. Then the matrix $V^{t} C V$ in (3.2) is positive semidefinite if and only if $S^{t} V^{t} C V S$ is positive definite where $S$ is an invertible matrix. In particular, let $S=D^{-1} P$ where $D=\operatorname{diag}\left(1, \sqrt{\lambda_{1}}, \cdots, \sqrt{\lambda_{k}}, 1, \ldots, 1\right)$ and $P$ is an orthogonal matrix so that the first two rows of $P^{t}$ are

$$
(1,0, \ldots, 0) \quad \text { and } \quad \frac{1}{\gamma}\left(0, \frac{b_{1}}{\sqrt{\lambda_{1}}}, \cdots, \frac{b_{k}}{\sqrt{\lambda_{k}}}, 0, \ldots, 0\right)
$$

respectively, where $\gamma=\left\{\sum_{j=1}^{k} b_{j}^{2} / \lambda_{j}\right\}^{1 / 2}$. Then

$$
\lambda P^{t} D^{-1} V^{t} C V D^{-1} P=\left(\begin{array}{cc}
n \lambda-b_{0} & -\gamma \\
-\gamma & 1
\end{array}\right) \oplus I_{k-1} \oplus 0_{n-k-1}
$$

which is positive semidefinite if and only if $\lambda \geq\left(b_{0}+\sum_{j=1}^{k} b_{k}^{2} / \lambda_{j}\right) / n$. It follows that $\lambda C=\lambda e e^{t}-A$ is positive semidefinite and $C$ is a correlation matrix whenever $\lambda \geq\left(b_{0}+\sum_{j=1}^{k} b_{k}^{2} / \lambda_{j}\right) / n$.

By the argument in the preceding paragraph, we see that whenever

$$
A=\left(\left\|x_{i}-x_{j}\right\|^{2}\right)=\left(\left\|x_{i}\right\|^{2}+\left\|x_{j}\right\|^{2}-2 x_{i}^{t} x_{j}\right)=\lambda\left(e e^{t}-\left(x_{i}^{t} x_{j} /\left\|x_{i}\right\|\left\|x_{j}\right\|\right)\right)
$$

with $r=\left\|x_{1}\right\|=\cdots=\left\|x_{n}\right\|$, then $\lambda=2 r^{2} \geq\left(b_{0}+\sum_{j=1}^{k} b_{k}^{2} / \lambda_{j}\right) / n$. The last assertion follows.

$(h) \Leftrightarrow(d)$. The equivalence of $(\mathrm{h})$ and $(\mathrm{d})$ is clear. 
$(h) \Leftrightarrow(e)$. Let $L$ denote the leading $(k+1) \times(k+1)$ principal submatrix of $V^{t} A V$. Because $\lambda_{1}, \ldots, \lambda_{k}>0, L$ has at least $k$ negative eigenvalues. But trace $(L)=$ $\operatorname{trace}\left(V^{t} A V\right)=\operatorname{trace}(A)=0$, so $b_{0}>0$ and $L$ must have a positive eigenvalue. Thus, $\operatorname{rank}(L)=k+1$ and $\operatorname{rank}(A)=\operatorname{rank}\left(V^{t} A V\right) \geq k+1$. It is obvious from the form of (3.1) that $\operatorname{rank}(A)=\operatorname{rank}\left(V^{t} A V\right)>k+1$ if and only if some $b_{i} \neq 0$, $i \in\{k+1, \ldots, n-1\}$.

$(h) \Leftrightarrow(f)$. Let $V$ be the orthogonal matrix in (h). Then that $e^{t} w=1$ if and only if $V^{t} w=\left(1, w_{2}, \ldots, w_{n}\right)^{t}$ for some $w_{2}, \ldots, w_{n} \in \Re$. So, (f) holds if and only if $b_{k+1}=\cdots=b_{n}=0$.

$(d) \Leftrightarrow(g)$. It is easy to show that (d) and (g) are equivalent.

$(h) \Leftrightarrow(c)$. Using the matrix representation in (h), we see that the intersection of the range space of $A$ and the null space of $\left(I-e e^{t} / n\right) A\left(I-e e^{t} / n\right)$ contains the vector $e$. It has dimension one if and only if $b_{k+1}=\cdots=b_{n}=0$.

(b) $\Rightarrow$ (i). Note that for $\lambda \geq 0$ and correlation matrix $C$, that $-\lambda y^{t} C y \leq 0$. It follows that $\sum a_{i j} y_{i} y_{j}=y^{t} A y=\lambda y^{t} e e^{t} y-\lambda y^{t} C y \leq \lambda\left(\sum y_{i}\right)^{2}$. Thus, condition (i) holds with $M=\lambda$.

$$
\text { (i) } \Rightarrow(f) \text {. If there exists an M such that } \sum_{i, j=1}^{n} a_{i j} y_{i} y_{j} \leq M\left(\sum_{i=1}^{n} x_{i}\right)^{2} \text { for all } y \in
$$
$\mathbb{R}^{n}$, then restrict $y$ to those values such that $\sum y_{i}=e^{t} y=1$. Then $y^{t} A y \leq M<\infty$ for all such $y$.

The equivalence of (a) and (e) was observed by Gower [7, Theorem 6]. The equivalence of (a) and (d) of Theorem 3.1 and the last assertion were obtained in [12, Theorem 3.1 and Corollary 3.1].

Alfakih and Wolkowicz [1, Theorem 3.3] used Gale transforms to characterize those ESD matrices that can be represented as $A=\lambda\left(e e^{t}-C\right)$. In particular, they state condition (h) as $A Z=0$, where $Z$ is a Gale matrix associated with $A$. If $k=n-1$, then $Z=(0, \ldots, 0)^{t}$ and $A Z=0$, while (h) is vacuously true. If $k<n-1$, then let $Z=\left(u_{k+1} \cdots u_{n-1}\right) Q$, where $u_{1}, \ldots, u_{n-1}$ are the columns of $U$ and $Q$ is nonsingular. Then $U^{t} A Z=0$ and, because the rows of $U^{t}$ form a basis for $e^{\perp}$, the columns of $A Z$ lie in the span of $e$. Hence, $A Z=0$ if and only if $\left(b_{k+1}, \ldots, b_{n-1}\right)^{t}=$ $e^{t} A Z=0$.

Using condition (b), one can deduce that $\operatorname{CESD}(n)$ is a convex cone. Note that it is highly non-trivial to prove that $\operatorname{CESD}(n)$ is a convex cone using the definition. 
The next result is noted in [5, p. 535], as a corollary of an elegant but complicated general theory of cuts and metrics. Here we provide a direct proof and some refinements.

Theorem 3.2. The set $\operatorname{CESD}(n)$ is dense in $\operatorname{ESD}(n)$, i.e., $\operatorname{ESD}(n)$ equals the closure of $\operatorname{CESD}(n)$.

Proof. Suppose $A \in \operatorname{ESD}(n)$ has rank $m$. If $A$ has embedding dimension $m-1$, then $A \in \operatorname{CESD}(n)$. Otherwise, let $A=\left(\left\|x_{i}-x_{j}\right\|^{2}\right)$ such that $x_{1}, \ldots, x_{n} \in \mathbb{R}^{m-2}$ so that $\sum_{j=1}^{n} x_{j}=0$. Let $X$ be the $(m-2) \times n$ matrix with $x_{1}, \ldots, x_{n}$ as column. Then there is an $(n-m+1) \times n$ matrix so that the columns of $X^{t}$ and $Y^{t}$ together form an orthonormal basis for $e^{\perp}$. For $\varepsilon>0$, let $Z_{\varepsilon}$ be the $(n-1) \times n$ matrix with the top $m-2$ rows from the matrix $X$, and the bottom $n-m+1$ rows from the matrix $\varepsilon Y$. Let $y_{1}, \ldots, y_{n} \in \mathbb{R}^{n-m+1}$ be the column of $Y$. Then

$$
A_{\varepsilon}=\kappa\left(Z_{\varepsilon}^{t} Z_{\varepsilon}\right)=\left(\left\|x_{i}-x_{j}\right\|^{2}\right)+\varepsilon^{2}\left(\left\|y_{i}-y_{j}\right\|^{2}\right)
$$

has embedding dimension $n-1$, and hence belongs to $\operatorname{CESD}(n)$. Evidently, $A_{\varepsilon}$ approaches $A$ as $\varepsilon \rightarrow 0$.

4. Linear preservers. In this section, we study linear maps leaving invariant the cones of $\operatorname{ESD}(n)$ and $\operatorname{CESD}(n)$. Actually, using the correspondence between positive semidefinite matrices, ESD matrices and CESD matrices, we can obtain better results. We begin by characterizing the linear operators that preserve PSD matrices with specific ranks.

TheOrem 4.1. Let $K=\left\{k_{1}, \ldots, k_{m}\right\} \neq\{0\}$ be such that $0 \leq k_{1}<\ldots<k_{m} \leq$ $n-1$. Let

$$
\mathcal{C}=\{C \in \operatorname{PSD}(n-1): \operatorname{rank}(C) \in K\} .
$$

Then a linear operator $T: \mathbf{S}_{n-1} \rightarrow \mathbf{S}_{n-1}$ satisfies $T(\mathcal{C})=\mathcal{C}$ if and only if there exists an invertible matrix $R$ such that

$$
T(C)=R^{t} C R \quad \text { for all } C \in \mathbf{S}_{n-1} .
$$

Proof. If $T(C)=R^{t} C R$ with $R$ invertible, then it follows from Sylvester's Law of Inertia that $T(C)$ has the same number of positive, negative and zero eigenvalues, i.e. is PSD of the same rank. Thus we see that $T(\mathcal{C})=\mathcal{C}$. It remains to establish the converse. Let

$$
\mathcal{C}_{j}=\{C \in \operatorname{PSD}(n-1): \operatorname{rank}(C)=j\} \quad \text { and } \quad \hat{\mathcal{C}}_{k}=\bigcup_{j=0}^{k} \mathcal{C}_{j}
$$


We claim that $\hat{\mathcal{C}}_{k}=\operatorname{cl}\left(\mathcal{C}_{k}\right)$, the closure of $\mathcal{C}_{k}$.

Because $\mathcal{C}_{k} \subseteq \hat{\mathcal{C}}_{k}$ and $\hat{\mathcal{C}}_{k}$ is closed, we see that $\operatorname{cl}\left(\mathcal{C}_{k}\right) \subseteq \hat{\mathcal{C}}_{k}$. If $C \in \mathcal{C}_{k}$, then obviously $C \in \operatorname{cl}\left(\mathcal{C}_{k}\right)$. If $C \in \mathcal{C}_{j}$ for $j<k$, then write $C=Y_{j}^{t} Y_{j}$ for a $j \times(n-1)$ matrix $Y_{j}$. Let $Y_{k-j}$ be any $(k-j) \times(n-1)$ matrix such that $\left(Y_{j}^{t} \mid Y_{k-j}^{t}\right)$ has rank $k$, let $c=1 /\left\|Y_{k-j}^{t} Y_{k-j}\right\|$, and let $C_{i}=Y_{j}^{t} Y_{j}+(c / i) Y_{k-j}^{t} Y_{k-j}$. Then $C_{i} \in \mathcal{C}_{k}$ and $\left\|C_{i}-C\right\|=1 / i \rightarrow 0$ as $i \rightarrow \infty$, so each $C \in \hat{\mathcal{C}}_{k}$ is the limit point of a sequence in $\hat{\mathcal{C}}_{k}$. This proves that $\hat{\mathcal{C}}_{k} \subseteq \operatorname{cl}\left(\mathcal{C}_{k}\right)$. It also demonstrates that $\operatorname{int}\left(\hat{\mathcal{C}}_{k}\right)$, the relative interior of $\hat{\mathcal{C}}_{k}$, is contained in $\mathcal{C}_{k}$. Because $\mathcal{C}_{k} \subseteq \hat{\mathcal{C}}_{k}$ and $\hat{\mathcal{C}}_{k}$ is closed, $\operatorname{cl}\left(\mathcal{C}_{k}\right) \subseteq \hat{\mathcal{C}}_{k}$. We have also shown that because $\mathcal{C}_{k}$ is open in $\hat{\mathcal{C}}_{k}, \operatorname{int}\left(\hat{\mathcal{C}}_{k}\right)=\mathcal{C}_{k}$.

Now suppose that $T(\mathcal{C})=\mathcal{C}$. Because $T$ is continuous,

$$
T\left(\hat{\mathcal{C}}_{k_{m}}\right)=T(\operatorname{cl}(\mathcal{C}))=\operatorname{cl}(\mathcal{C})=\hat{\mathcal{C}}_{k_{m}}
$$

Because $T$ is linear,

$$
T\left(\mathcal{C}_{k_{m}}\right)=T\left(\operatorname{int}\left(\hat{\mathcal{C}}_{k_{m}}\right)\right)=\operatorname{int}\left(\hat{\mathcal{C}}_{k_{m}}\right)=\mathcal{C}_{k_{m}} .
$$

From (4.3) and (4.2), we obtain

$$
T\left(\hat{\mathcal{C}}_{k_{m}-1}\right)=T\left(\hat{\mathcal{C}}_{k_{m}}-\mathcal{C}_{k_{m}}\right)=T\left(\hat{\mathcal{C}}_{k_{m}}\right)-T\left(\mathcal{C}_{k_{m}}\right)=\hat{\mathcal{C}}_{k_{m}}-\mathcal{C}_{k_{m}}=\hat{\mathcal{C}}_{k_{m}-1} .
$$

We continue to "peel the onion" in this manner, i.e., using an inductive argument, concluding that $T\left(\mathcal{C}_{1}\right)=\mathcal{C}_{1}$. It then follows from Theorem 3 in [8] that $T$ is of the form $T(C)= \pm R^{t} C R$. Because $C$ and $T(C)$ are positive semidefinite, we conclude that $T(C)=R^{t} C R$.

Next we set $w=e$ and characterize the linear operators that preserve subsets of $G_{e}(n)$ containing matrices with specific ranks.

TheOREM 4.2. Let $K=\left\{k_{1}, \ldots, k_{m}\right\} \neq\{0\}$ be such that $0 \leq k_{1}<\ldots<k_{m} \leq$ $n-1$. Let

$$
\mathcal{B}=\left\{B \in G_{e}(n): \operatorname{rank}(B) \in K\right\} .
$$

Then a linear operator $T:\left[G_{e}(n)\right] \rightarrow\left[G_{e}(n)\right]$ satisfies $T(\mathcal{B})=\mathcal{B}$ if and only if there exists an $n \times n$ matrix $Q$, with $\operatorname{rank}(Q)=n-1$ and $Q e=Q^{t} e=0$, such that

$$
T(B)=Q^{t} B Q \quad \text { for all } B \in\left[G_{e}(n)\right] .
$$

Proof. Fix $w=e$ and $U$, any $n \times(n-1)$ matrix for which $\left(\frac{e}{\sqrt{n}} \mid U\right)$ is orthogonal. Then $W=U^{t}\left(I-\frac{e e^{t}}{n}\right)=U^{t}$, so $\psi_{u}(B)=U^{t} B U$ and $\phi_{u}(C)=W^{t} C W=U C U^{t}$. 
Let $\mathcal{C}=\psi_{u}(\mathcal{B})$, in which case $\mathcal{B}=\phi_{u}(\mathcal{C})$. Then $T(\mathcal{B})=\mathcal{B}$ if and only if $T \circ \phi_{u}(\mathcal{C})=$ $\phi_{u}(\mathcal{C})$ if and only if $\psi_{u} \circ T \circ \phi_{u}(\mathcal{C})=\mathcal{C}$. Because $\psi_{u}$ and $\phi_{u}$ preserve rank, $\mathcal{C} \subseteq$ $\operatorname{PSD}(n-1)$ is a set of the form (4.1); hence, it follows from Theorem 4.1 that $T(\mathcal{B})=\mathcal{B}$ if and only if there exists an invertible matrix $R$ such that $\psi_{u} \circ T \circ \phi_{u}(C)=R^{t} C R$.

Suppose that there exists an $(n-1) \times(n-1)$ invertible matrix $R$ such that $\psi_{u} \circ T \circ \phi_{u}(C)=R^{t} C R$. Let $Q=U R U^{t}$. Then $\operatorname{rank}(Q)=n-1, Q e=Q^{t} e=0$, and

$$
\begin{aligned}
T(B) & =\phi_{u} \circ \psi_{u} \circ T \circ \phi_{u} \circ \psi_{u}(B)=\phi_{u} \circ \psi_{u} \circ T \circ \phi_{u}\left(U^{t} B U\right) \\
& =\phi_{u}\left(R^{t} U^{t} B U R\right)=U R^{t} U^{t} B U R U^{t}=Q^{t} B Q .
\end{aligned}
$$

Conversely, suppose that there exists an $n \times n$ matrix $Q$ such that $\operatorname{rank}(Q)=n-1$, $Q e=Q^{t} e=0$, and $T(B)=Q^{t} B Q$. Let $R=U^{t} Q U$. Then $R$ is invertible and $\psi_{u} \circ T \circ \phi_{u}(C)=\psi_{u} \circ T\left(U C U^{t}\right)=\psi_{u}\left(Q^{t} U C U^{t} Q\right)=U^{t} Q^{t} U C U^{t} Q U=R^{t} C R$.

Now, we characterize the linear operators that preserve matrices in $\operatorname{ESD}(n)$ with specific embedding dimensions. Let $\operatorname{dim}(A)$ denote the embedding dimension of $A \in$ $\operatorname{ESD}(n)$.

TheOrem 4.3. Let $K=\left\{k_{1}, \ldots, k_{m}\right\} \neq\{0\}$ be such that $0 \leq k_{1}<\ldots<k_{m} \leq$ $n-1$. Let

$$
\mathcal{A}=\{A \in \operatorname{ESD}(n): \operatorname{dim}(A) \in K\} .
$$

Then a linear operator $T:[\operatorname{ESD}(n)] \rightarrow[\operatorname{ESD}(n)]$ satisfies $T(\mathcal{A})=\mathcal{A}$ if and only if there exists an $n \times n$ matrix $Q$, with $\operatorname{rank}(Q)=n-1$ and $Q e=Q^{t} e=0$, such that

$$
T(A)=-\kappa\left(Q^{t} A Q\right) / 2 \quad \text { for all } A \in[\operatorname{ESD}(n)] .
$$

Proof. Let $\mathcal{B}=\tau_{e}(\mathcal{A})$, in which case $\mathcal{A}=\kappa(\mathcal{B})$. Then $T(\mathcal{A})=\mathcal{A}$ if and only if $T \circ \kappa(\mathcal{B})=\kappa(\mathcal{B})$ if and only if $\tau_{e} \circ T \circ \kappa(\mathcal{B})=\mathcal{B}$. Because of the equivalence of conditions (a) and (b) in Theorem 2.1, $\mathcal{B} \subseteq G_{e}(n)$ is a set of the form (4.4); hence, it follows from Theorem 4.2 that $T(\mathcal{A})=\mathcal{A}$ if and only if there exists an $n \times n$ matrix $Q$, with $\operatorname{rank}(Q)=n-1$ and $Q e=Q^{t} e=0$, such that

$$
\tau_{e} \circ T \circ \kappa(B)=Q^{t} B Q .
$$

Now we apply $\kappa$ to both sides of (4.5), obtaining

$$
\begin{aligned}
T(A) & =T \circ \kappa(B)=\kappa\left(Q^{t} B Q\right)=\kappa\left(Q^{t} \tau_{e}(A) Q\right) \\
& =-\frac{1}{2} \kappa\left(Q^{t}\left(I-\frac{e e^{t}}{n}\right) A\left(I-\frac{e e^{t}}{n}\right) Q\right)=-\frac{1}{2} \kappa\left(Q^{t} A Q\right) .
\end{aligned}
$$


By the fact that the closure of $\operatorname{CESD}(n)$ equals $\operatorname{ESD}(n)$, we have the following.

Theorem 4.4. A linear operator $T:[\operatorname{CESD}(n)] \rightarrow[\operatorname{CESD}(n)]$ satisfies

$$
T(\operatorname{CESD}(n))=\operatorname{CESD}(n)
$$

if and only if there exists an $n \times n$ matrix $Q$, with $\operatorname{rank}(Q)=n-1$ and $Q e=Q^{t} e=0$, such that

$$
T(A)=-\kappa\left(Q^{t} A Q\right) / 2 \quad \text { for all } A \in[\operatorname{CESD}(n)] \text {. }
$$

\section{REFERENCES}

[1] A. Y. Alfakih and H. Wolkowicz. Two theorems on Euclidean distance matrices and Gale transform. Linear Algebra and Its Applications, 340:149-154, 2002.

[2] L. M. Blumenthal. Theory and Applications of Distance Geometry. Clarendon Press, Oxford, 1953.

[3] A. Cayley. On a theorem in the geometry of position. Cambridge Mathematics Journal, 2:267271, 1841. Attribution: "From a Correspondent.".

[4] G. M. Crippen and T. F. Havel. Distance Geometry and Molecular Conformation. John Wiley \& Sons, New York, 1988.

[5] M. M. Deza and M. Laurent. Geometry of Cuts and Metrics. Springer, Berlin, 1997.

[6] J. C. Gower. Some distance properties of latent root and vector methods in multivariate analysis. Biometrika, 53:325-338, 1966.

[7] J. C. Gower. Properties of Euclidean and non-Euclidean distance matrices. Linear Algebra and Its Applications, 67:81-97, 1985.

[8] J. W. Helton and L. Rodman. Signature preserving linear maps of Hermitian matrices. Linear and Multilinear Algebra, 17(1):29-37, 1985.

[9] C.-K. Li and S. Pierce. Linear preserver problems. American Mathematical Monthly, 108(7):591-605, 2001

[10] S. W. Malone, P. Tarazaga, and M. W. Trosset. Better initial configurations for metric multidimensional scaling. Computational Statistics and Data Analysis, 41(1):143-156, 2002.

[11] M. W. Richardson. Multidimensional psychophysics. Psychological Bulletin, 35:659-660, 1938. Abstract of presentation at the forty-sixth annual meeting of the American Psychological Association, September 7-10, 1938.

[12] P. Tarazaga, T. L. Hayden, and J. Wells. Circum-Euclidean distance matrices and faces. Linear Algebra and Its Applications, 232:77-96, 1996.

[13] W. S. Torgerson. Multidimensional scaling: I. Theory and method. Psychometrika, 17:401-419, 1952.

[14] G. Young and A. S. Householder. Discussion of a set of points in terms of their mutual distances. Psychometrika, 3:19-22, 1938. 